\title{
Urban-rural Linkages and Spatial-temporal Land Use Change in Beijing-Tianjin-Hebei Metropolitan Region: A New Perspective
}

\author{
Yuheng Li \\ PhD student of Urban and Regional Studies, Department of Urban Planning and Environment \\ School of Architecture and Built Environment, KTH (Royal Institute of Technology), Stockholm, Sweden
}

Received: May 15, 2011

Accepted: May 29, 2011

doi:10.5539/jsd.v4n4p61

Sponsoring information: The written of this paper is supported by China Scholarship Council (No. 2008635048).

\begin{abstract}
The paper aims to investigate land use change at the county level in Beijing-Tianjin-Hebei Metropolitan Region. Three land use patterns are identified by assessing the urban-rural linkages: urban, peri-urban and periphery areas. Empirical analysis shows that land use change in the urban areas is faster than that in the peri-urban and periphery areas. Economic conditions are significant to the land use change in the urban areas while the accessibility is the major driving force to the land use change in the peri-urban areas. However, weak factor influence is found in the periphery areas. Basically, the differences of land use change and the driving factors imply how the resource flows and agglomerates in this region. The paper finally emphasizes the role of peri-urban areas as the interface between urban and periphery areas for coordinating land use this region in the future.
\end{abstract}

Keywords: Urban-rural linkages, Land use change, Spatial-temporal, Principal component analysis, Beijing-Tianjin-Hebei

\section{Introduction}

China has experienced accelerated urbanization since 1978. Its urbanization level (ratio of people living in the cities to the total population) increased from 17.9\% in 1978 to $45.7 \%$ in 2008 (National Bureau of Statistics of China, 2009). In this process, cities underwent fast growth due to the prosperous economy and population increase. As Fan (1999) identified, Chinese cities have over the past several decades, dramatically experienced two-dimension growth: vertically, existing cities of different size have expanded both in population and land area; horizontally, a large number of newly designated cities have been added to the existing urban system. The direct consequence of such urban growth is the continuous increase of built land and the decrease of arable land.

Beijing-Tianjin-Hebei Metropolitan Region (Jing-Jin-Ji for short. Jing, Jin and Ji are the abbreviations of Beijing, Tianjin and Hebei Province) is one of the regions in China experiencing fast urbanization development and land use change. Urbanization level in this region increased from $7.4 \%$ in 1990 to $49 \%$ in 2000 (National Bureau of Statistics of China, 1991 and 2001). In the same period, arable land in this region decreased by $2178.5 \mathrm{~km}^{2}$ while built land increased by $1721.5 \mathrm{~km}^{2}$ (Liu et al. 2005a, b). Many studies have analyzed land use change in this region. Tan, et al. (2005) investigated the differences of urban land expansion and arable land loss among different-tier cities in Jing-Jin-Ji region. They found enormous differences of urban land development among the different-tier cities, and around 74\% of new urban land comes from arable land conversion. Wang, et al. (2008) analyzed the pattern of cultivated land conversion to built land in Jing-Jin-Ji region. They found that the outspread of built land in Beijing, Tianjin and Hebei Province decreased from 1995 to 2000 comparing with that in the period 1985-1995. Xu (2008) and $\mathrm{Li}$, et al. (2009) examined the built land change in Beijing, Tianjin and other eight provincial cities in Hebei Province. Differences of built land change among these cities are revealed.

Generally, current studies made detailed analysis of land use change in Jing-Jin-Ji region. However, they merely examined land use change at the city level as a whole. Since each city consists of many counties and towns, thus, the studies didn't adequately show the land use change at a lower level (counties or urban districts). Besides, land use change differentiates in different places due to the demographic and economic conditions. For example, places adjacent to the urban areas are easily to be converted to the construction land due to the urban expansion while distant areas may have slow land use change. However, such land use differences were not given particular attention in the current studies. Thus, the paper attempts to investigate land use change at the county level in Jing-Jin-Ji region and examine the differences of land use change in different places. Unlike previous studies, the paper tries to bring land use change and resource flows into the analysis framework. The point of departure is that places of different resource agglomerations have different land use change. The second section reviews the relation between land use change and urban-rural resource flows. In section three, the paper assesses urban-rural 
linkages and divides this region according to the assessment. Then, the paper examines land use change and its driving factors in the divisions. Finally, the paper concludes through discussing the research findings.

\section{Theoretical framework: urban-rural linkages and land use pattern}

Basically, land use change is closely related to the human-nature interactions and comprises the dynamic relations between human activities and physical environment. Krausmann, et al. (2004) described the relation between land use change and the socio-economic material and energy flows as: area is needed to extract materials and energy from the environment to harbor the infrastructure, to transport, transform, store, and consume materials and energy, and to deposit or absorb wastes. Mitsuda and Ito (2011) also argued that socio-economic factors which captured the human-induced land conditions are dominating land use change among different land use patterns. Thus, land use change emerges because of the human-induced resource flows.

Generally, urban and rural areas are tightly connecting with each other. This connection displays as resource flows of people, capital, information, goods and technology between them. Potter, et al. (2004) argued that urban-rural linkages were initiated in an attempt to make use of the mutual differentials or complementarities. The central place is supplying high-order services to the surrounding areas which in turn provide low-order services like food and other resource to the central place (Christaller, 1933). The core-periphery theory (Friedmann, 1966) described the unequal distribution of power in economy, society and polity between the core and periphery areas. The core area dominates the central realm while the surrounding rural periphery is dependent on the central place for the supply of high-order services. Finally, the core area evolves into urban or metropolitan with high potential for innovation and growth while periphery areas experience slow growth or even stagnation, adding its dependency on the core area. Krugman (1991) and Fujita, et al. (1999) argued that core-periphery economy may emerge due to the interrelated industry concentrations, reliable infrastructure and high accessibility to the market. Barkley, et al. (1999) pointed out that the agglomeration effect in the central places can be strengthened when educated laborers were attracted and superior infrastructure was constructed. Thus, resource flows tends to agglomerate to the urban areas while the fortunes of rural areas are tightly relied on the adjacent urban areas.

Recently, much attention has been paid to the urban-rural interface. McGee (1991) used 'desakota' in the Asian context to describe the symbiosis of urban and rural areas which resulted from the transformation into a dispersed metropolis. Gu, et al. (1995) considered urban fringe as a new and independent object between cities and villages. Gering, et al. (1998) labeled the urban-rural interface and found it a zone where social, economic and political factors interact in complex ways. These manifestations all emphasized the transitional and dynamic features of urban-rural interface which serves as a frontier where rural areas are under transition to urban areas. Urban-rural interface acts as an attractive destination for rural migrants, offering non-farm employment and access to education, medical service. The role of urban-rural interface has also been recognized when the out-migration and industrial transfer from downtown to the outskirts and suburbs emerged because of the congestion problems in the urban areas. Krugman and Elizondo (1996) indicated that resource relocating in the urban-rural interface is attributed to the "centrifugal" forces that tend to break the agglomerations in the urban areas.

Based on the above analysis, three resource agglomeration patterns can be drawn. The first is the non-agricultural activities agglomerating to the urban areas which have high profit returns, accessible services and large market. Built land develops quickly in this pattern due to the continuous resource flows e.g. migration. The second pattern is the peri-urban areas, serving as the urban-rural interface and providing services, industrial production and employment to the rural areas. Arable land at peri-urban areas is easily to convert for non-agricultural use. Situating between cities and countryside, land use in peri-urban areas possess both the urban and rural attributes. The third pattern is found in the rural periphery which is dominated by agricultural production, supplying food and other materials to the urban areas.

Basically, the three resource agglomeration patterns also applied at the regional level in China. Years of urbanization development since the reform and opening-up in 1978 has seen continuous and large scale resource flows into the urban areas. Take the rural-urban migration for example, in the period 1978-1999, the annual average number of rural-urban migrants is 7.1 million contributing to $72.7 \%$ of annual urban population growth in China (National Bureau of Statistics of China, 1999). However, due to the congestion problems like pollution and land price increase, counter-urbanization also emerged in large cities of China in the late 1980s. The downtown areas of Beijing, Shanghai, Shenyang and Dalian saw population decrease $(-3.4 \%,-2.3 \%,-6.7 \%$ and $-11.8 \%)$ in the period $1982-1990$ while tremendous population increase $(40.5 \%, 55.5 \%, 31 \%$ and $56 \%)$ took place in the suburbs of these cities (Zhou and Meng, 1998). Besides, the development of township and village enterprises which are expected to attract more rural peasants so as to prohibit immigrants in the big cities have contributed to the development of small and medium cities and towns. In the period 1991-1999, the number of small cities (population below 0.2 million) increased from 297 to 365, an increase of $22.9 \%$ (National Bureau of Statistics of China, 1992-2000). Thus, land use change in the urban areas, small towns and the countryside would differentiate due to the different resource agglomerations at the regional level in the Chinese context. 


\section{Arable and built land use change in Jing-Jin-Ji region}

\subsection{Study area}

Jing-Jin-Ji region (183000 $\mathrm{km}^{2}$ ) includes Beijing, Tianjin (directly-governed cities under jurisdiction of central government) and eight prefecture-level cities in Hebei Province (Zhang Jiakou, Chengde, Qin Huangdao, Tangshan, Langfang, Cangzhou, Baoding and Shi Jiazhuang) (Figure 1). Beijing is the capital of China and Tianjin is the third largest city in China. Hebei is located in the Huabei Plain of China and surrounding Beijing and Tianjin. In 2008, there were 78.6 million people in this region producing $9.9 \%$ of the GDP in China (National Bureau of Statistics of China, 2009).

[Figure 1 probably here]

\subsection{Methodology and data}

Tight connection between urban-rural linkages and land use change is shown in section two. The paper hypothesizes that land use change differentiates due to the differences of resource agglomerations in the three resource agglomeration patterns. Besides, the driving factors to the land use change are also different in the three patterns. To examine this hypothesis, the paper tries to assess urban-rural linkages and examine land use change through dividing Jing-Jin-Ji region according to the assessment.

Tacoli (1998) has made detailed analysis of rural-urban linkages in terms of flows of people, goods and wastes as well as the related flows of information and money across space between urban and rural areas. Although these flows are in a complex process making it hard to monitor, one consequence of the resource flows is that both urban and rural areas are affected by the transformations at the macro-level including demographic and economic changes. Given the data availability, the paper selects six variables representing the demographic and economic changes and indirectly assesses urban-rural linkages of each county (or urban district) in Jing-Jin-Ji region.

(1) Increase of percentage of urban population to the total population. This variable is selected to show the rural-urban population mobility in the urbanization process.

(2) Increase of percentage of non-agricultural employees to the total employees. This variable is chosen to show the change of employment structure (employees in the primary, secondary and tertiary industries).

(3) Increase of non-agricultural production. This variable is selected to show the development of non-agricultural industries. Particularly, the development of non-agricultural industries in rural areas contributes a lot to the non-agricultural production in China.

(4) Increase of rural household per capita net income. This variable is chosen to show the change of rural household income due to the sectoral interactions which have diversified the rural economy and rural households' income.

(5) Increase of per capita GDP. This variable is selected to describe the economic growth both in urban and rural areas due to the mutual resource flows.

(6) Increase of rural electricity consumption (kwh). This variable is chosen to show the development of non-agricultural industries in rural areas because of their sectoral interactions with the cities.

Considering the correlation among the variables, the paper uses principal component analysis $(P C A)$ to transform these correlated variables into a smaller number of uncorrelated variables which are called principal components. This method can reduce data dimensionality through covariance analysis between factors.

Suppose there are $m$ variables $X_{1} \ldots X_{m}$ and $m$ new variables can be generated through $P C A$, known as the principal components (PC), $P C_{1} \ldots P C_{m}$ which can be expressed as follows:

$$
\begin{aligned}
P C_{1} & =a_{11} X_{1}+\ldots+a_{1 m} X_{m}=X a_{1} \\
P C_{m} & =a_{m 1} X_{1}+\ldots+a_{m m} X_{m}=X a_{m}
\end{aligned}
$$

Where $a_{i}$ are the coefficients for PC, and each column of $a$ contains the coefficients for one PC. Here $P C_{1}$ and $P C_{2}$ which have the first and second largest variance are chosen on the condition that they are uncorrelated. If we consider that the sample variance-covariance matrix of the original variables $X$ is $N_{x}$, then, the coefficient vector $a_{i}$ can be computed through the equation:

$$
\left|N_{x}-\lambda I\right| a=0
$$

Where $\lambda$ is the vector of characteristic roots and $a$ is a matrix comprising of the characteristic vectors corresponding to each characteristic root. It is noted that $P C_{1}$ is computed by using the characteristic vector corresponding to the largest characteristic root $\lambda_{1}$ while $P C_{2}$ is computed by using the characteristic vector corresponding to the largest characteristic root $\lambda_{2}$, and so on (Harris, 2001). 
Remote sensing data is used in this paper to detect and monitor land use change in Jing-Jin-Ji region between 1990 and 2000. This data is from the Chinese National Land Cover Database (CNLCD) and developed by Chinese Academy of Sciences (Liu, et al., 2005a, b). A hierarchical classification system of 25 land cover classes was aggregated to arable land, urban \& rural built land and others in this study. In this database, Jing-Jin-Ji region was divided into 109 counties and 10 urban districts. Thus, the economic and demographic data for all the units in this region also follows this division. The economic and demographic data comes from the Hebei Economic Yearbook, Beijing Statistical Yearbook and Tianjin Statistical Yearbook in the same calendar year of 1991 and 2001.

3.3 Results and interpretation

According to the conventional rule of extracting components which have eigenvalues greater than one, two components were extracted by PCA, accounting for $81.6 \%$ of the variance (Table 1). The first component which explains $63.3 \%$ of the variance is the most important component among the variables. This component includes five variables except the increase of rural household per capita net income which belongs to the second component (Table 1).

[Table 1 probably here]

The sub-model for each component was made according to the component coefficients.

$$
\begin{gathered}
F_{1}=0.31 X_{1}+0.31 X_{2}+0.50 X_{3}+0.49 X_{4}+0.08 X_{5}+0.42 X_{6} \\
F_{2}=0.43 X_{1}-0.19 X_{2}-0.18 X_{3}+0.19 X_{4}+0.83 X_{5}+0.17 X_{6}
\end{gathered}
$$

Two component scores of units in Jing-Jin-Ji region were computed through Formula 1 and 2 and presented in Table 2 and Table 3. These scores are uncorrelated and representing the selected six variables. These scores are used as the base for cluster analysis through which group memberships are assigned to all the units. The paper uses PASW Statistics18 and conducts K-Means cluster analysis for each urban administration (Beijing, Tianjin and other eight cities in Hebei). This analysis reveals a natural break that divides all the units within each urban administration into three classifications (Figure 2).

\section{[Table 2 probably here] \\ [Table 3 probably here] \\ [Figure 2 probably here]}

The first classification consists of urban districts of Beijing, Tianjin, Shi Jiazhuang, Chengde, Zhang Jiakou and Baoding as well as their close counties. Thus, this classification is named "urban areas". The second classification as shown in Figure 2, includes counties which are mainly surrounding units of the first part and it is named "peri-urban areas". The third classification covers the countryside and the distant counties in each city administration and is named "periphery areas".

The three parts identified by the cluster analysis also differ in the demographic and economic conditions (Table 4). Urban areas have the largest population size in 2000 and highest annual demographic increase in the period 1990-2000. Periphery areas have the second largest population size in 2000, but its annual population growth is lowest among the three classifications. Annual population increase in the peri-urban areas belongs to the medium level. In the economic domain, urban areas have high level of per capita GDP (2000) followed by peri-urban and periphery areas. However, annual per capita GDP increase in periphery areas is $9.7 \%$ which is higher than that in peri-urban areas $(8.7 \%)$ and urban areas $(7.6 \%)$.

\section{[Table 4 probably here]}

\subsubsection{The change of arable land and built land in the three classifications}

In the period 1990-2000, arable land in Jing-Jin-Ji region decreased by $2178.5 \mathrm{~km}^{2}$ with the annual decrease of $0.26 \%$ while built land increased by $1721.5 \mathrm{~km}^{2}$ with the annual increase of $1.3 \%$. Figure 3 shows the land distribution in Jing-Jin-Ji region in 1990 and 2000. Generally, the increase of built land mainly takes place in the urban areas of Beijing, Tianjin, Shi Jiazhuang and Tangshan as well as their surrounding areas. However, the northern and western parts of this region which are mainly mountainous areas display little land change in this period.

[Figure 3 probably here]

To show the differences of change of arable land and built land in the three classifications, the paper designates $\mathrm{R}$ to denote the relative changing rate of a certain land type in a period. $\mathrm{R}$ can be expressed as:

$$
R=\left(\left|N_{b}-N_{a}\right| * M_{a}\right) /\left(\left|M_{b}-M_{a}\right| * N_{a}\right)
$$


In this formula, $N_{a}$ and $N_{b}$ are the size of a certain land type in a certain area at the beginning and the end of the study period; $M_{a}$ and $M_{b}$ are the size of a certain land type in the whole region at the beginning and the end of the study period.

Table 5 presents the computing results of the average relative land change in each classification in the period 1990-2000. The figures clearly show the differences of land change among the three classifications. Both arable land and built land in the urban areas have the highest changing rates, followed by the peri-urban areas and the periphery areas. This indicates that dramatic land change mainly takes place in the urban areas and their surrounding areas while the distant places have slow land change. Given the demographic and economic differences shown in Table 4, the difference of land change also implies that areas of fast population increase and high economic level usually experience large scale and fast land change. Besides, this result is in line with the hypothesis of the study: the change of arable land and built land in the three urban-rural interaction patterns are different.

[Table 5 probably here]

3.2.2 Driving factors to the land change in Jing-Jin-Ji region

This section makes regression analysis to further analyze the driving factors to the change of arable land and built land in the three classifications in Jing-Jin-Ji region. The paper chooses three types of factors:

(1) Demographic factors. Initial population density $\left(D_{90}\right)$ of each unit and its increase $\Delta D$ are selected to show the influence of initial population and its increase on the land change.

(2) Economic factors. Per capita GDP in $1990\left(P g d p_{90}\right)$, its increase from 1990 to 2000 ( $\triangle P g d p$ ), the ratio of non-agricultural industrial employment to the total employment in $1990\left(\mathrm{Na}_{90}\right)$ and its increase in the period 1990-2000 ( $\Delta N a)$ are chosen to show the influence of economy and industrial structure on the land change.

(3) Geographical factors. The paper selects the initial highway mileage per square kilometer $\left(R_{90}\right)$ and its increase $(\Delta R)$ from 1990 to 2000 to represent the infrastructure conditions. Accessibility Index is also chosen to show the accessibility (e.g. access to market and finance) of each unit in this region. The paper holds that accessibility contributes to the resource flows and agglomeration in each unit. The formula can be written (Note $1)$ :

$$
A c_{i}=\sum P_{i} / d_{i j}{ }^{\beta} \quad(j, j \neq i)
$$

$A c_{i}$ is the aggregate accessibility index of $\mathrm{i} t h$ unit (i=1,2,3..129), $P_{j}$ is the population size of the $\mathrm{j} t h$ central city, $d_{i j}$ is the distance between $\mathrm{i}$ th unit and $\mathrm{j} t h$ central city $(\mathrm{j}=1,2,3 \ldots 10)$. Concerning the population change of each central city, the paper uses the variation of this index in the period 1990-2000. $A c_{90}$ is the accessibility of all the units in 1990. $\triangle A c$ is accessibility change in this period.

$L_{90}$ and $L_{00}$ are the land size (arable land and built land) in 1990 and 2000. Then, the regression model can be written:

$$
\ln L_{00}-\ln L_{90}=\alpha+\beta\left(D_{90}, \Delta D\right)+\theta\left(P g d p_{90}, \Delta P g d p, N a_{90}, \Delta N a\right)+\gamma\left(R_{90}, \Delta R, A c_{90}, \Delta A c\right)+\varepsilon
$$

A correlation analysis was made before the regression to test for autocorrelations among the variables. Those of high correlations with other variables will be removed from the regression in each classification. Results in Table 6 show that the driving factors to the changes of arable land and built land in the three classifications are different. Generally, the increase of per capita GDP is the sole and significant factor, contributing to the arable land decrease and built land increase in the urban areas. In the peri-urban areas, the increase of accessibility is the most significant factor while the initial population density makes contribution to the land use change in this classification. Besides, arable land decrease in the peri-urban areas is also attributed to the increase of per capita GDP and highway mileage per square kilometer in 1990. The increase of accessibility is significant to the arable land decrease in the periphery areas while all the variables show no significance to the built land increase in this classification. Basically, regression results indicate that economic conditions are dominating land use change in the urban areas while the accessibility (e.g. market, services) is influencing land use change in the peri-urban areas. However, rather weak influence is found in the periphery areas. This difference also implies that high economic level attracts resource to the urban areas while high accessibility makes it possible for the resource (rural resource and the diffusing from urban) agglomerating to the peri-urban areas. Resource outflow or limited resource agglomeration makes land use change in the periphery areas at a low level.

$$
\text { [Table } 6 \text { probably here] }
$$

\section{Concluding remarks}

The paper examined the arable and built land use change at the county level in Jing-Jin-Ji region. Three urban-rural linkage patterns were identified by assessing urban-rural linkages of all the counties/urban districts in 
this region: urban, peri-urban and periphery areas. Research findings show that the changes of arable land and built land in the urban areas are faster than that in the peri-urban and periphery areas. Besides, the driving factors to the land use change in these linkage patterns are also found different. Basically, the research of the paper revealed the relation between land use change and urban-rural resource flows in the Chinese context. Comparing with previous studies, the paper divided Jing-Jin-Ji region in terms of the urban-rural resource flows and agglomeration instead of administrations. Thus, how the resource flows and agglomerates at the regional level can be implied based on the changes of arable land and built land. The major lessen of the research is that it is not enough to just analyze land use change at the city level as a whole. For various counties or towns, land use change is different due to the differences of resource flows and agglomeration in different places.

The research also helps to predict the future land use change in Jing-Jin-Ji region. Due to the fast socio-economic development, built land in the urban areas will gain expansion continuously and will definitely induce the decrease of arable land. However, land use change in the periphery areas will stay at a low level because of the limited resource flows and agglomeration. Thus, the coordination of built land expansion in the urban areas and land development in the periphery areas will challenge the regional planning in the future. The paper revealed the medium-level of resource flows and agglomeration in the peri-urban areas. Thus, situating between urban and rural areas, peri-urban areas can help to provide hinterlands for the urban areas and boost development in the periphery areas. In this sense, the importance of peri-urban areas as the urban-rural interface lies in that it can distract the excessive resource agglomeration in the urban areas, and bring the urban services to the periphery areas at the same time.

Generally, the research of the paper was conducted in a region which has been experiencing fast urbanization development. In this process, urban-rural resource flows such as rural-urban migration and economic activities contribute to the land use change in urban, rural and peri-urban areas. Thus, this research methodology can be an example for similar studies of land use change in other regions which are experiencing fast urbanization development and intense urban-rural resource flows.

\section{Notes}

1. According to the index, it is supposed to compute the aggregated accessibility of all the units to each unit. Nevertheless, the paper holds that migration to each unit is mainly dominated by its accessibility to the central cities. Thus, the paper computes the aggregated accessibility of each unit to the ten central cities (Beijing, Tianjin and eight prefecture-level cities in Hebei Province).

\section{References}

Barkley, D. Henry, M. and Kim, Y. (1999). Industry Agglomerations and Employment Change in Non-metropolitan Areas. Review of Urban and Regional Development Studies. (11), 168-186, doi: 10.1111/1467-940X.00014

Beijing Municipal Bureau of Statistics. (1991). Beijing statistical yearbook. Beijing: China Statistic Press.

Beijing Municipal Bureau of Statistics. (2001). Beijing statistical yearbook. Beijing: China Statistic Press.

Christaller, W. (1933). Die zentralen Orte in Süddeutschland (The central places in Southern Germany).Jena: Verlag von Gustav Fischer.

Fan, C.C. (1999). The Vertical and Horizontal Expansions of China's City System. Urban Geography. 20(6), 493-515, doi:10.2747/0272-3638.20.6.493

Friedmann, J. (1966). Regional development policy-A case stuay of Venezuela. Cambridge: MIT Press.

Fujita, M., Krugman, P., \& Venables, A. (1999). The spatial economy: Cities, regions and international trade. Cambridge: MA: MIT Press.

Gering, L.R., Chun, A.V. \& Anderson, S. (1998). Defining and predicting urban-wild land interface zones using a GIS-based model. Boise, Idaho, USA, Forest Service General Technical Report NC-212, August 16-20.

Gu, C.L., Ding, J.H., Chen, T., et al. (1995). Research on China urban fringe. Beijing: Science Press.

Harris, J.R. (2001). A primer of multivariate statistics ( $3^{\text {rd }}$ edition). London: Lawrence Erlbaum Associates Publishers.

Hebei Provincial Bureau of Statistics. (1991). Hebei economic yearbook. Beijing: China Statistic Press.

Hebei Provincial Bureau of Statistics. (2001). Hebei economic yearbook. Beijing: China Statistic Press.

Krausmann, F., Haberl, H. and Erb, K.H. et al. (2004). Resource Flows and Land Use in Austria 1950-2000: Using the MEFA Framework to Monitor Society-Nature Interaction for Sustainability. Land Use Policy. 21(3), 215-230, doi:10.1016/j.landusepol.2003.10.005

Krugman, P. (1991). Increasing Returns and Economic Geography. Journal of Political Economy. 99(3), 483-499,

http://links.jstor.org/sici?sici=0022-3808\%28199106\%2999\%3A3\%3C483\%3AIRAEG\%3E2.0.CO\%3B2-6\&ori gin=repec 
Krugman, P. and Elizondo, R. L. (1996). Trade Policy and the Third World Metropolis. Journal of Development Economics, 49(1), 137-150, http://EconPapers.repec.org/RePEc:eee:deveco:v:49:y:1996:i:1:p:137-150

Li, X.J. and Lian, J. et al. (2009). Spatial and Temporal Analysis for Urban and Rural Construction Land Use of Beijing-Tianjin-Hebei Metropolis Circle. Urban Remote Sensing Joint Event. 1-5, doi: 10.1109/URS.2009.5137613

Liu, J.Y., et al. (2005a). Spatial and Temporal Patterns of China's Cropland During 1990-2000: An Analysis Based on Landsat TM data. Remote Sensing of Environment. 98(4), 442-456, doi:10.1016/j.rse.2005.08.012

Liu, J.Y., et al. (2005b). China's Changing Landscape During the 1990s: Large-scale Land Transformations Estimated with Satellite Data. Geophysical Research Letters. 32 (L02405), 1-5, doi: 10.1029/2004GL021649

McGee, T.G. (1991). The emergence of Desakota regions in Asia: Expanding a hypothesis. In N. Ginsburg, B. Koppel, and T.G. McGee (Eds.). The extended metropolis: Settlement transition in Asia (pp. 3-25). Honolulu: University of Hawaii Press.

Mitsuda, Y. and Ito. S. (2011). A Review of Spatial-Explicit Factors Determining Spatial Distribution of Land use/Land-use Change. Landscape and Ecological Engineering. 7(1):117-125, doi: 10.1007/s11355-010-0113-4

National Bureau of Statistics of China (2009). Comprehensive statistical data and materials on 50 years of new China. Beijing: China Statistic Press.

National Bureau of Statistics of China (2009). China statistical yearbook. Beijing: China Statistic Press.

National Bureau of Statistics of China (1991-2001). China statistical yearbook. Beijing: China Statistic Press.

Potter, R. \& Binns, T. et al. (2004). Geographies of development (2nd edition). Pearson: Harlow.

Tacoli, C. (1998). Rural-urban Interactions: A Guide to the Literature. Environment and Urbanization, 10(1), 147-166, doi: 10.1177/095624789801000105

Tan, M.H., and Li, X.B. et al. (2005). Urban Land Expansion and Arable Land Loss in China- a Case Study of Beijng-Tianjin-Hebei region. Land Use Policy. 22(3), 187-196, doi:10.1016/j.landusepol.2004.03.003

Tianjin Statistical Bureau (1991). Tianjin statistical yearbook. Beijing: China Statistic Press.

Tianjin Statistical Bureau (2001). Tianjin statistical yearbook. Beijing: China Statistic Press.

Wang, L., and Li, F.Y. et al. (2008). Cultivated Land Conversion Pattern and Impact Factors in Urban Agglomeration: A Case Study on Urban Agglomeration of Beijing,Tianjin and Hebei Province (in Chinese). China Land Science. (1), 32-38, doi: CNKI: SUN: ZTKX. 0. 2008-01-005

$\mathrm{Xu}$, H. (2008).Urban and Rural Built Land Change in Beijing-Tianjin-Hebei Metropolitan Region: Analysis Based on Remote Sensing and GIS', Master thesis (in Chinese), Capital Normal University, Beijing. May 17.

Zhou, Y.X. and Meng, Y.C. (1998). The Tendency of Suburbanization of big cities in China (in Chinese). Urban Planning Forum, 3, 22-27, doi: CNKI:SUN:CXGH.0.1998-03-004

Table 1. Rotated component matrix

\begin{tabular}{|c|c|c|}
\hline \multirow{2}{*}{ Selected variables } & \multicolumn{2}{|c|}{ Component } \\
\hline & Factor 1 & Factor 2 \\
\hline Increase of per capita GDP & 0.594 & 0.452 \\
\hline Increase of percentage of non-agricultural employees to the total employees & 0.594 & -0.195 \\
\hline Increase of non-agricultural production & 0.965 & 0.185 \\
\hline Increase of percentage of urban population to the total population & 0.954 & -0.196 \\
\hline Increase of rural household per capita net income & 0.157 & 0.865 \\
\hline Increase of rural electricity consumption & 0.816 & 0.182 \\
\hline Initial Eigenvalues & 3.800 & 1.096 \\
\hline$\%$ of Variance & 63.34 & 18.27 \\
\hline
\end{tabular}


Table 2. First component scores of units in Jing-Jin-Ji region

\begin{tabular}{|c|c|c|c|c|c|c|c|c|c|}
\hline Units & Score & Units & Score & Units & Score & Units & Score & Units & Score \\
\hline Beijing & 12.08 & Zanhuang & -0.93 & Lulong & -0.58 & Zhangiiakou & -0.26 & Cangxian & 1.50 \\
\hline Changping & 0.49 & Wuji & -0.46 & Baoding & 0.73 & Xuanhua & -0.87 & Qingxian & 0.53 \\
\hline Shunyi & 0.40 & Pingshan & -0.58 & Mancheng & -0.50 & Zhangbei & -1.14 & Dongguang & 0.36 \\
\hline Tongxian & -0.65 & Yuanshi & -0.55 & Qingyuan & -0.74 & Kangbao & -1.19 & Haixing & -0.28 \\
\hline Daxing & -0.12 & Zhaoxian & -0.44 & Dingzhou & $\begin{array}{l}-0.39 \\
\end{array}$ & Guyuan & -1.24 & Yanshan & 0.16 \\
\hline Pinggu & -0.49 & Xinji & 0.19 & Zhuozhou & 0.05 & Shangyi & -1.29 & Xiaoning & 0.06 \\
\hline Huairou & 0.24 & Gaocheng & 0.34 & Anguo & -0.34 & Weixian & -0.91 & Nanpi & 0.16 \\
\hline Miyun & -0.24 & Jinzhou & 0.02 & Gaobeidian & -0.05 & Yangyuan & -1.07 & Wuqiao & -0.04 \\
\hline Yanqing & -0.53 & Xinle & 0.15 & Yixian & $\begin{array}{l}-0.89 \\
\end{array}$ & Huaian & -1.21 & Xianxian & 0.56 \\
\hline Tianjin & 7.28 & Tangshan & 0.54 & Xushui & -0.39 & Wanquan & -1.27 & Mengcun & -0.32 \\
\hline Ninghe & 0.44 & Fengrun & 0.04 & Laiyuan & -1.02 & Huailai & -0.69 & Botou & 0.64 \\
\hline Wuqing & -0.20 & Fengnan & 1.17 & Dingxing & -0.52 & Zhuolu & -1.06 & Renqiu & 2.29 \\
\hline Jinghai & -0.48 & Luanxian & -0.02 & Shunping & -0.79 & Chicheng & -1.08 & Huanghua & 1.09 \\
\hline Baodi & -0.13 & Luannan & 0.21 & Tangxian & -1.03 & Chongli & -1.10 & Hejian & 1.32 \\
\hline Jixian & -0.24 & Leting & 0.43 & Wangdu & -0.96 & Chengde & -0.61 & Langfang & 0.99 \\
\hline $\begin{array}{c}\text { Shi } \\
\text { Jiazhuang }\end{array}$ & 1.48 & Qianan & 0.70 & Laishui & -1.13 & Chengdexian & -1.09 & Gu'an & 0.20 \\
\hline Jingxing & -0.33 & Qianxi & -0.26 & Gaoyang & -0.58 & Xinglong & -1.05 & Yongqing & -0.03 \\
\hline Huolu & 0.93 & Yutian & 0.13 & Anxin & -0.59 & Pingquan & -1.16 & Xianghe & 1.30 \\
\hline Zhengding & 0.50 & Tanghai & -0.01 & Xongxian & -0.63 & Luanping & -0.98 & Dacheng & 0.14 \\
\hline Luancheng & 0.27 & zunhua & 0.54 & Rongcheng & -0.56 & Longhua & -1.08 & Wenan & 0.55 \\
\hline Xingtang & -0.73 & $\begin{array}{c}\text { Qin } \\
\text { Huangdao }\end{array}$ & 0.38 & Quyang & -0.96 & Fengning & 0.19 & Dachang & 1.01 \\
\hline Lingshou & -0.69 & Qinglong & -1.19 & Fuping & -1.00 & Kuancheng & -0.31 & Bazhou & 1.69 \\
\hline Gaoyi & -0.26 & Changli & -0.53 & Boye & -1.06 & Weichang & 0.31 & Sanhe & 2.59 \\
\hline Shenze & -0.60 & Funing & -0.48 & Lixian & -0.28 & Cangzhou & 0.74 & & \\
\hline
\end{tabular}

Table 3. Second component scores of units in Jing-Jin-Ji region

\begin{tabular}{|c|c|c|c|c|c|c|c|c|c|}
\hline Units & Score & Units & Score & Units & Score & Units & Score & Units & Score \\
\hline Beijing & 6.56 & Zanhuang & -0.36 & Lulong & -0.27 & Zhangiiakou & -0.50 & Cangxian & 0.07 \\
\hline Changping & -0.18 & Wuji & 0.20 & Baoding & 0.43 & Xuanhua & -0.45 & Qingxian & -0.07 \\
\hline Shunyi & -0.26 & Pingshan & -0.24 & Mancheng & 0.09 & Zhangbei & -0.58 & Dongguang & -0.77 \\
\hline Tongxian & -0.63 & Yuanshi & -0.31 & Qingyuan & -0.12 & Kangbao & -0.59 & Haixing & -0.82 \\
\hline Daxing & -0.75 & Zhaoxian & 0.85 & Dingzhou & 0.38 & Guyuan & $\begin{array}{l}-0.61 \\
\end{array}$ & Yanshan & -0.77 \\
\hline Pinggu & -0.56 & Xinji & 0.77 & Zhuozhou & 0.007 & Shangyi & -0.63 & Xiaoning & -0.43 \\
\hline Huairou & -0.31 & Gaocheng & 1.37 & Anguo & -0.25 & Weixian & -0.51 & Nanpi & -0.81 \\
\hline Miyun & -0.45 & Jinzhou & 0.91 & Gaobeidian & 0.03 & Yangyuan & -0.54 & Wuqiao & -0.58 \\
\hline Yanqing & -0.50 & Xinle & 0.82 & Yixian & -0.44 & Huaian & -0.64 & Xianxian & -0.70 \\
\hline Tianjin & 2.68 & Tangshan & 0.61 & Xushui & -0.13 & Wanquan & -0.56 & Mengcun & -0.49 \\
\hline Ninghe & 0.25 & Fengrun & 0.92 & Laiyuan & -0.54 & Huailai & -0.41 & Botou & -0.59 \\
\hline Wuqing & 1.99 & Fengnan & 4.03 & Dingxing & -0.36 & Zhuolu & -0.53 & Renqiu & 0.42 \\
\hline Jinghai & 0.91 & Luanxian & 0.57 & Shunping & -0.19 & Chicheng & -0.57 & Huanghua & -0.38 \\
\hline Baodi & 0.003 & Luannan & 0.07 & Tangxian & -0.37 & Chongli & -0.57 & Hejian & 0.25 \\
\hline Jixian & -0.009 & Leting & 0.33 & Wangdu & -0.57 & Chengde & -0.47 & Langfang & -0.60 \\
\hline $\begin{array}{c}\text { Shi } \\
\text { Jiazhuang }\end{array}$ & 0.35 & Qianan & 1.19 & Laishui & -0.64 & Chengdexian & -0.41 & Gu'an & -0.33 \\
\hline Jingxing & -0.09 & Qianxi & -0.06 & Gaoyang & -0.10 & Xinglong & -0.54 & Yongqing & -0.62 \\
\hline Huolu & 1.58 & Yutian & 0.66 & Anxin & -0.25 & Pingquan & -0.38 & Xianghe & -0.38 \\
\hline Zhengding & 2.40 & Tanghai & 0.21 & Xongxian & 0.09 & Luanping & -0.41 & Dacheng & -0.06 \\
\hline Luancheng & 0.03 & zunhua & 0.41 & Rongcheng & -0.34 & Longhua & -0.47 & Wenan & -0.29 \\
\hline Xingtang & -0.17 & $\begin{array}{c}\text { Qin } \\
\text { Huangdao }\end{array}$ & -0.31 & Quyang & -0.53 & Fengning & -0.91 & Dachang & -0.17 \\
\hline Lingshou & 0.09 & Qinglong & -0.49 & Fuping & -0.55 & Kuancheng & -0.89 & Bazhou & 1.40 \\
\hline Gaoyi & -0.05 & Changli & -0.35 & Boye & -0.46 & Weichang & -0.95 & Sanhe & 0.23 \\
\hline Shenze & -0.24 & Funing & 0.07 & Lixian & 0.92 & Cangzhou & -0.47 & & \\
\hline
\end{tabular}


Table 4. Demographic and economic features in the three classifications in Jing-Jin-Ji region, 1990-2000

\begin{tabular}{|c|c|c|c|}
\hline Division & Urban areas & Peri-urban areas & Periphery areas \\
\hline Number of units & 28 & 37 & 54 \\
\hline Total population in 2000 (million) & 29.48 & 17.99 & 23.44 \\
\hline Annual population increase (\%) & 2.11 & 0.73 & 0.59 \\
\hline Per capita GDP in 2000 (¥/person) & 19715 & 9563 & 7509 \\
\hline Annual per capita GDP increase (\%) & 7.55 & 8.71 & 9.69 \\
\hline
\end{tabular}

Table 5. Average relative change of arable land and built land in Jing-Jin-Ji region, 1990-2000

\begin{tabular}{|c|c|c|c|c|}
\hline \multirow{2}{*}{ Division } & \multicolumn{2}{|c|}{ Arable land } & \multicolumn{2}{c|}{ Built land } \\
\cline { 2 - 5 } & $\begin{array}{c}\text { Change amount } \\
\left(\mathrm{km}^{2}\right)\end{array}$ & $\begin{array}{c}\text { Average relative } \\
\text { changing rate }(\%)\end{array}$ & $\begin{array}{c}\text { Change amount } \\
\left(\mathrm{km}^{2}\right)\end{array}$ & $\begin{array}{c}\text { Average relative } \\
\text { changing rate }(\%)\end{array}$ \\
\hline Urban areas & -824.17 & 2.06 & 881.70 & 1.52 \\
\hline Peri-urban areas & -651.61 & 1.01 & 583.31 & 1.30 \\
\hline Periphery areas & -702.71 & 0.62 & 256.48 & 0.37 \\
\hline
\end{tabular}

Table 6. Regression results of driving factors to the land use change in Jing-Jin-Ji region, 1990-2000

\begin{tabular}{|c|c|c|c|c|c|c|c|c|c|c|c|}
\hline Divisions & $D_{90}$ & $\Delta D$ & $P g d p_{90}$ & $\Delta P g d p$ & $N a_{90}$ & $\Delta N a$ & $R_{90}$ & $\Delta R$ & $A c_{90}$ & $\Delta A c$ & $\mathrm{R}^{2}$ \\
\hline $\begin{array}{l}\text { Urban } \\
\text { arable }\end{array}$ & - & - & - & $\begin{array}{c}-0.63 \\
(-3.48)^{* *}\end{array}$ & - & $\begin{array}{c}0.28 \\
(1.46)\end{array}$ & - & $\begin{array}{c}-0.006 \\
(-0.034)\end{array}$ & - & $\begin{array}{l}0.039 \\
(0.23)\end{array}$ & 0.60 \\
\hline $\begin{array}{c}\text { Peri-urban } \\
\text { arable }\end{array}$ & $\begin{array}{c}-0.24 \\
(-1.79)^{*}\end{array}$ & - & - & $\begin{array}{c}-0.27 \\
(-2.11)^{*}\end{array}$ & - & $\begin{array}{c}0.14 \\
(1.19)\end{array}$ & $\begin{array}{c}0.30 \\
(2.18)^{*}\end{array}$ & - & - & $\begin{array}{c}-0.58 \\
(-4.81)^{* *}\end{array}$ & 0.79 \\
\hline $\begin{array}{c}\text { Periphery } \\
\text { arable }\end{array}$ & - & $\begin{array}{c}-0.088 \\
(-0.599)\end{array}$ & - & $\begin{array}{c}0.11 \\
(0.79)\end{array}$ & - & $\begin{array}{c}-0.21 \\
(-1.57)\end{array}$ & $\begin{array}{c}0.15 \\
(0.97)\end{array}$ & - & - & $\begin{array}{c}-0.47 \\
(-3.61)^{* *}\end{array}$ & 0.51 \\
\hline $\begin{array}{c}\text { Urban } \\
\text { built }\end{array}$ & - & - & - & $\begin{array}{c}0.56 \\
(2.93)^{* *}\end{array}$ & - & $\begin{array}{c}-0.17 \\
(-0.88)\end{array}$ & - & $\begin{array}{l}-0.054 \\
(-0.28)\end{array}$ & - & $\begin{array}{c}-0.12 \\
(-0.63)\end{array}$ & 0.54 \\
\hline $\begin{array}{c}\text { Peri-urban } \\
\text { built }\end{array}$ & $\begin{array}{c}0.29 \\
(1.89)^{*}\end{array}$ & - & - & $\begin{array}{l}0.088 \\
(0.60)\end{array}$ & - & $\begin{array}{c}-0.05 \\
(-0.38)\end{array}$ & $\begin{array}{c}-0.11 \\
(-0.70)\end{array}$ & - & - & $\begin{array}{c}0.57 \\
(4.10)^{* *}\end{array}$ & 0.70 \\
\hline $\begin{array}{c}\text { Periphery } \\
\text { built }\end{array}$ & - & $\begin{array}{l}0.046 \\
(0.28)\end{array}$ & - & $\begin{array}{l}0.097 \\
(0.60)\end{array}$ & - & $\begin{array}{l}0.064 \\
(0.44)\end{array}$ & $\begin{array}{l}-0.071 \\
(-0.40)\end{array}$ & 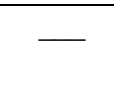 & - & $\begin{array}{c}0.23 \\
(1.54)\end{array}$ & 0.27 \\
\hline
\end{tabular}

Note: Figures in parenthesis are associated $t$ values. $*=$ sig. $<0.1, * *=$ sig. $<0.05$ 


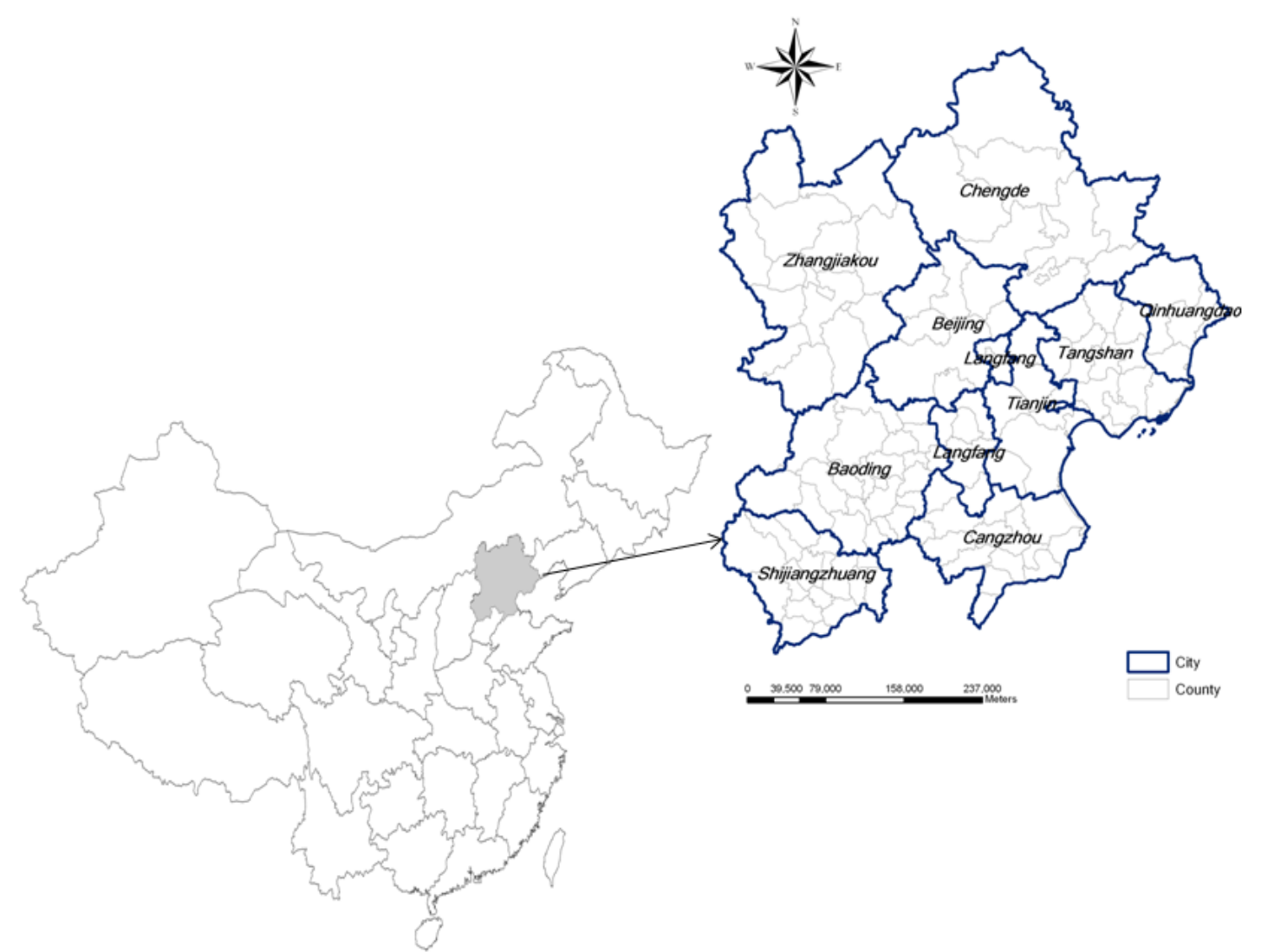

Figure 1. Ten cities and the counties in Jing-Jin-Ji region

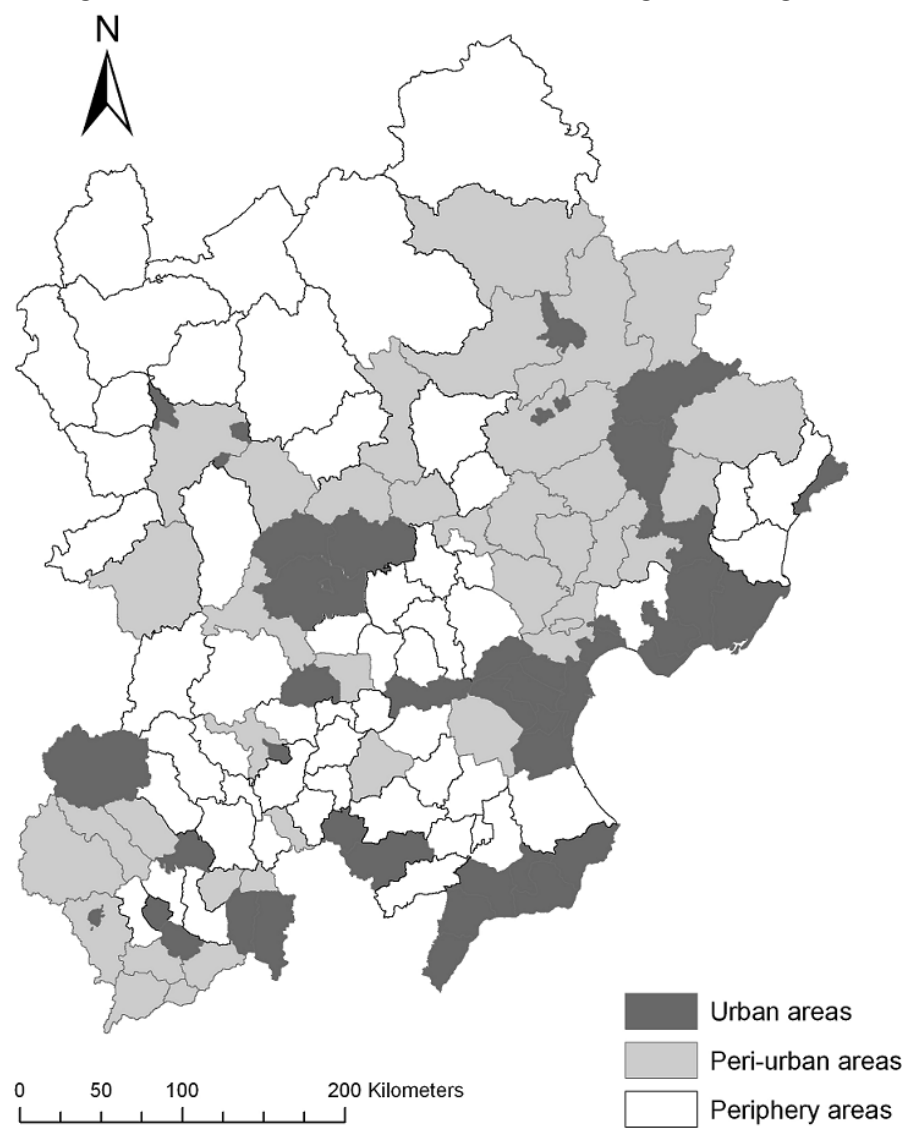

Figure 2. Three classifications by cluster analysis in Jing-Jin-Ji region 


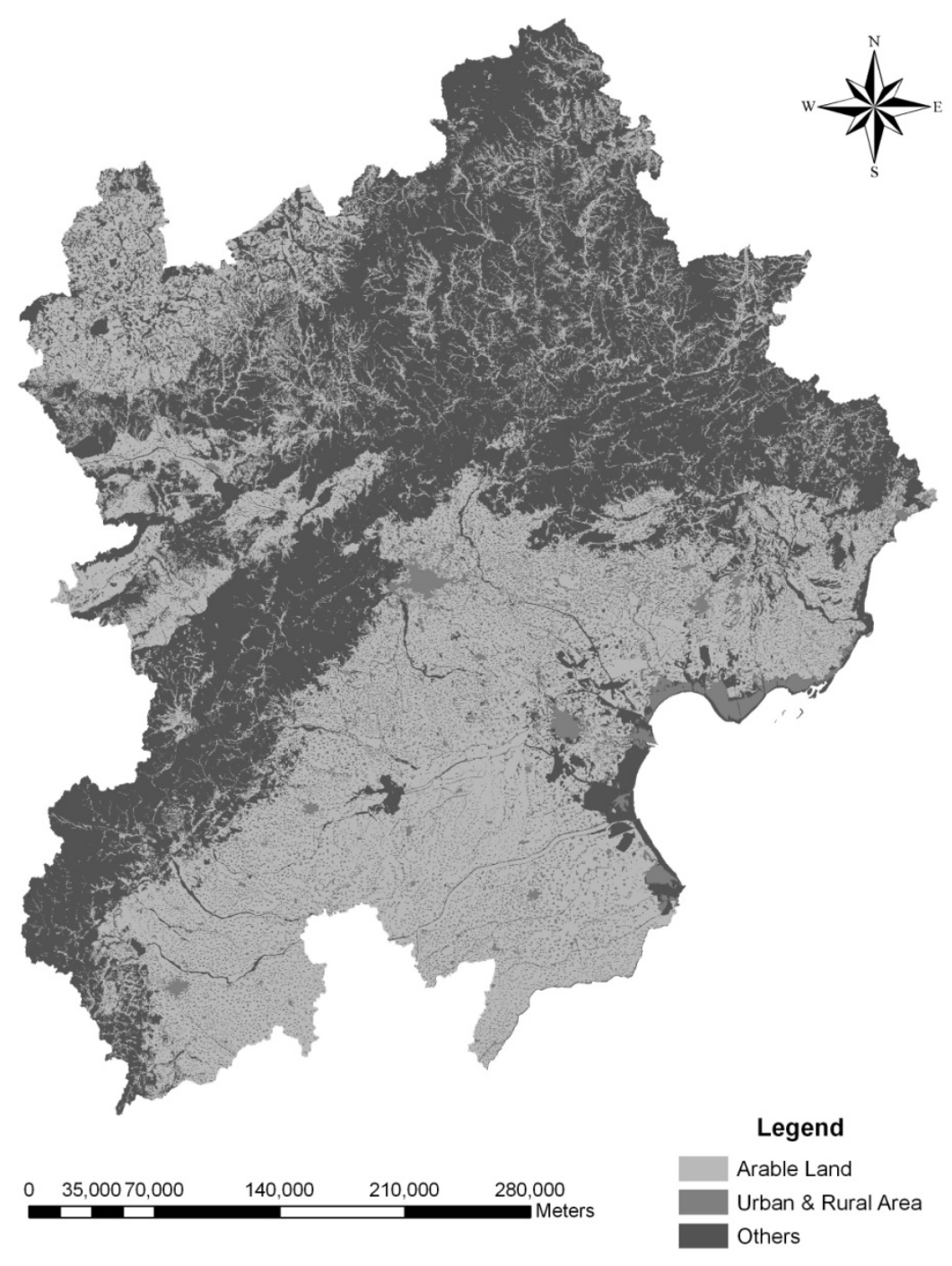

(1990) 


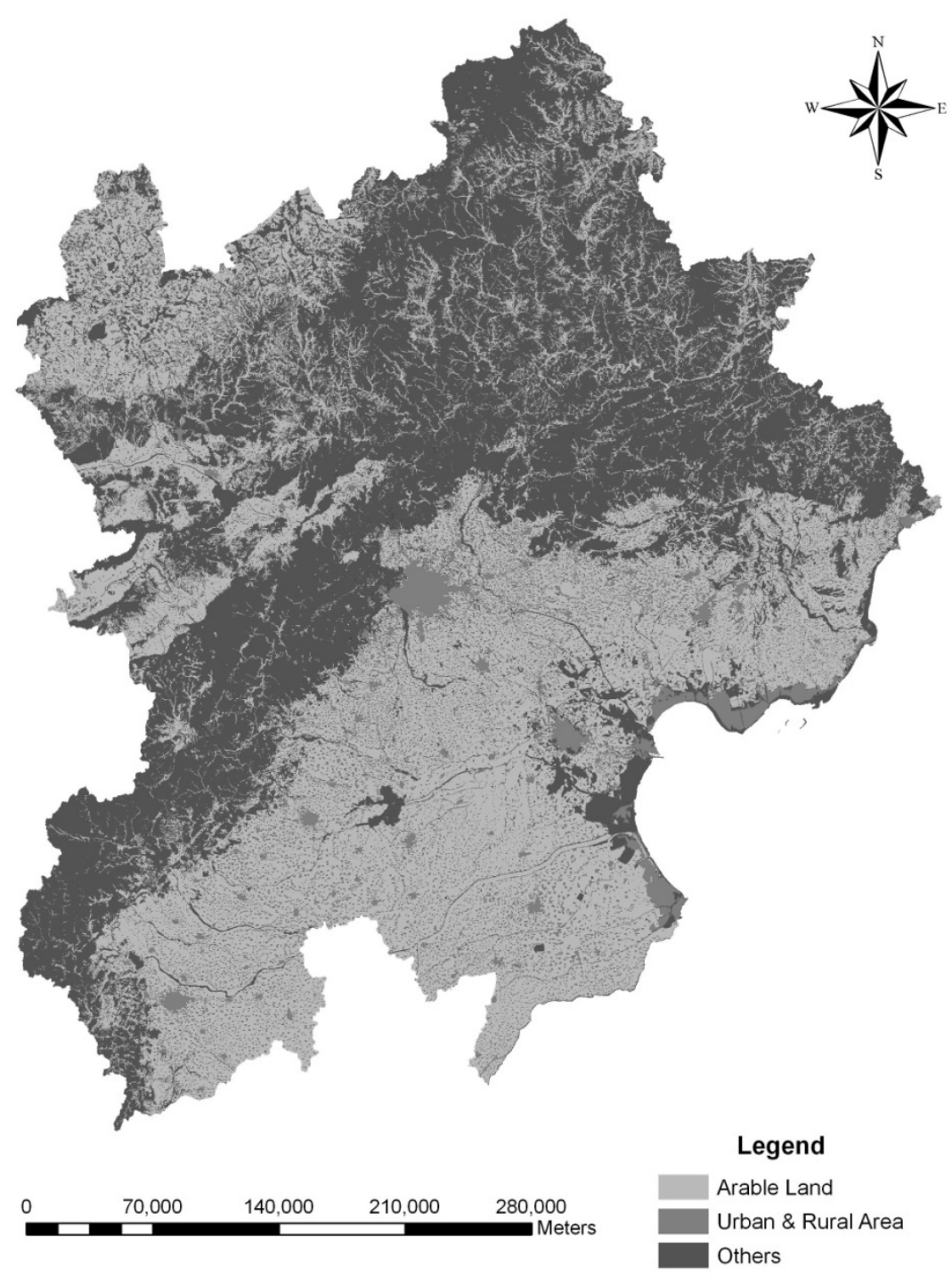

(2000)

Figure 3. Land use in Jing-Jin-Ji region, 1990 and 2000 